\title{
VIVÊNCIA DAS FAMÍLIAS E O CUSTO NO CUIDADO DE CRIANÇAS E ADOLESCENTES COM MIELOMENINGOCELE
}

\section{EXPERIENCE OF FAMILIES AND THE COST IN THE CARE OF CHILDREN AND ADOLESCENTS WITH MYELOMENINGOCELE}

\section{LA EXPERIENCIA DE LAS FAMILIAS Y EL COSTO EN EL CUIDADO DE NIÑOS Y ADOLESCENTES CON MIELOMENINGOCELE}

Giselle Lima de Freitas ${ }^{1}$, Fabiana Faleiros ${ }^{2}$, Kênia Lara Silva ${ }^{3}$.

\section{RESUMO}

Objetivo: Compreender as vivências das famílias e o custo ao cuidado de crianças e adolescentes com mielomeningocele. Método: Pesquisa exploratória realizada com 16 famílias de crianças e adolescentes com mielomeningocele usuários de um Hospital de Reabilitação de Belo Horizonte/MG. Para a coleta de dados, utilizaram-se a entrevista e a observação, durante as três visitas domiciliares, realizadas em cada família participante. Resultados: A renda familiar média foi de dois salários mínimos. $O$ baixo nível socioeconômico foi agravado pelas dificuldades à aquisição gratuita de materiais para a realização dos cuidados. Os custos diretos com os cuidados relacionaram-se à compra de medicamentos, fraldas, cateteres urinários e gastos com transporte, sendo menores para as famílias de adolescentes independentes. Conclusão: Os pais de crianças em condições crônicas vivenciam barreiras econômicas e sociais relacionadas ao custo do cuidado em domicílio e necessitam de suporte com serviços de atenção primária e de reabilitação.

Descritores: Meningomielocele; Efeitos Psicossociais da Doença; Enfermagem em Reabilitação.

\begin{abstract}
Objective: To understand the experience of families and the cost in the care of children and adolescents with myelomeningocele. Method: An exploratory research conducted with 16 families of children and adolescents with myelomeningocele users from a Rehabilitation Hospital in Belo Horizonte/MG. For data collection, interviews and observation were used during the three home visits to each participating family. Results: The average family income was about two minimum wages. The low socioeconomic level was aggravated by the difficulties in the free acquisition of materials to perform the care. The direct care costs were related to drug purchase, diapers, urinary catheters and transportation expenses, being lower for families of independent adolescents. Conclusion: Parents of children with chronic conditions experience economic and social barriers related to the cost of home care and need support with primary care and rehabilitation services.
\end{abstract}

Descriptors: Meningomyelocele; Psychosocial Effects of the Illness; Rehabilitation Nursing.

\section{RESUMEN}

Objetivo: Comprender la experiencia de las familias y el costo del cuidado a niños y adolescentes con mielomeningocele. Método: Una investigación exploratoria realizada con 16 familias con niños y adolescentes enfermos de mielomeningocele de un Hospital de Rehabilitación en Belo Horizonte/MG. Para la recolección de datos se utilizaron entrevistas y observación durante las tres visitas domiciliarias a cada familia participante. Resultados: El ingreso familiar promedio fue de aproximadamente 2 salarios mínimos. EI bajo nivel socioeconómico se vio agravado por las dificultades en la adquisición gratuita de materiales para realizar el cuidado. Los costos directos de la atención médica se relacionaron con la compra de medicamentos, pañales, sondas urinarias y gastos con transporte, siendo menores para las familias con adolescentes independientes. Conclusión: Los padres de niños con afecciones crónicas experimentan barreras económicas y sociales relacionadas con el costo de la atención domiciliaria y necesitan apoyo de los servicios de atención primaria y rehabilitación.

Descriptores: Meningomielocele; Efectos Psicosociales de la Enfermedad; Enfermería en Rehabilitación.

${ }^{1}$ Doutora em Enfermagem. Docente do Departamento de Enfermagem Materno Infantil e Saúde Pública da Universidade Federal de Minas Gerais. ${ }^{2}$ Doutora em Ciências da Reabilitação. Docente do Departamento de Enfermagem Geral e Especializada da Escola de Enfermagem de Ribeirão Preto da Universidade de São Paulo. ${ }^{3}$ Enfermeira. Doutora em Enfermagem. Docente do Departamento de Enfermagem Aplicada da Universidade Federal de Minas Gerais.

Como citar este artigo:

Freitas GL, Faleiros F, Silva KL. O custo do cuidado para famílias de crianças e adolescentes com mielomeningocele. Revista de Enfermagem do Centro Oeste Mineiro. 2019;9:e3282. [Access__]; Available in:__. DOI: http://dx.doi.org/10.19175/recom.v9i0.3282 


\section{INTRODUÇÃO}

A mielomeningocele (MMC) é a mais complexa má-formação dos defeitos de fechamento do tubo neural (DFTN) compatível com a vida e representa $75 \%$ de em pacientes acometidos $^{(1)}$. Os DFTN possuem multifatorialidade causal e constituem a segunda maior causa de má-formações congênitas, precedida apenas pelas cardíacas ${ }^{(2)}$. A carência de ácido fólico é identificada como o fator principal responsável pela ocorrência da doença, destacando-se, também, o diabetes materno, a deficiência de zinco, a ingestão de álcool, de anticonvulsivantes e de anestésicos ${ }^{(3)}$.

Aproximadamente 6000 crianças nasceram com MMC, na Europa, no período entre 1991 e 2011. Estima-se que a taxa de incidência mundial seja de 0,2 a 6,4/1000 nascidos vivos, observando-se valores mais altos nos países menos desenvolvidos ${ }^{(4)}$. Nos Estados Unidos, temse $0,34 / 1.000$ nascidos vivos ${ }^{(5)}$. A incidência de casos, no Brasil, é estimada em 0,83/1.000 a $1,87 / 1.000$ nascidos vivos, no entanto a real dimensão do problema permanece desconhecida, uma vez que não existe levantamento de abrangência nacional, e os estudos apontam para taxas divergentes ${ }^{(6)}$. Reconhece-se o aumento de sobrevida nesse público e a necessidade de compressão (ou compreensão?) das repercussões na qualidade de vida para a adolescência e a vida adulta $^{(4)}$.

O comprometimento motor e sensitivo varia de acordo com a localização - torácica, lombar ou sacral - e a gravidade da lesão espinhal(7). Como principais sequelas ou complicações neurológicas, tem-se a hidrocefalia, a má-formação de Arnold Chiari, as alterações cognitivas, a medula presa e a siringomielia ${ }^{(8)}$. As alterações urológicas e intestinais, também, exigem a necessidade de cuidados contínuos em domicílio, de natureza complexa e comprometem a qualidade de vida de indivíduos acometidos e suas famílias ${ }^{(9-10)}$. Ademais, crianças e adolescentes com MMC podem apresentar dificuldade de locomoção, deformidades musculoesqueléticas e alterações de sensibilidade cutânea ${ }^{(10)}$.

As sequelas e as complicações decorrentes da MMC impõem a necessidade de seguimento de um plano terapêutico direcionado aos diferentes sistemas corporais, acometidos pela má-formação, conferindo um caráter de cronicidade. A condição de cronicidade exige a continuidade do cuidado em domicílio e a necessidade de compra e manipulação de materiais, insumos e medicamentos relacionados ao cuidado, bem como ao gasto com transporte, para avaliações com diferentes profissionais ou atendimento de intercorrências clínicas $^{(9,11)}$. As adaptações, no cotidiano da criança e do adolescente com MMC e de sua família, são imperativas, provocando limitações empregatícias para os pais, que originam forte impacto nas atividades rotineiras, na realidade financeira da família e na qualidade de vida(11-12).

Os custos com o cuidado podem ser categorizados em diretos, indiretos e intangíveis ou emocionais. Os custos diretos relacionam-se à compra de materiais e insumos e despesas relacionadas ao transporte, à alimentação e ao tratamento. Os custos indiretos referem-se à perda ou à redução da capacidade laboral do indivíduo acometido ou de seu cuidador pela alta demanda e necessidade de cuidados. Integram os custos indiretos o recebimento de benefício pelo governo, o absenteísmo e a aposentadoria precoce por invalidez. E os custos intangíveis ou emocionais são os que repercutem nas condições psicológicas e inter-relacionais das crianças e de toda a família, causando dor e sofrimento ${ }^{(13-14)}$.

O custo com o cuidado de crianças e adolescentes com MMC aumenta as chances de vulnerabilidade social, interferindo em sua socialização com seus pares de idade e na rotina de toda a família(11). Os estudos brasileiros que abordam as vivências familiares e os custos nos cuidados de crianças e adolescentes com MMC são escassos ${ }^{(12)}$, assim, questiona-se qual a influência dos custos do cuidado no cotidiano da família de crianças e adolescentes com MMC? Diante deste contexto, este estudo teve como objetivo compreender as vivências das famílias e o custo para o cuidado de crianças e adolescentes com mielomeningocele.

\section{MÉTODOS}

Pesquisa exploratória, de abordagem qualitativa, que utilizou o referencial metodológico da dialética ${ }^{(15)}$, sustentado pelo referencial teórico do cotidiano de Agnes Heller $^{(16)}$. A adoção da dialética e de sua essência, a contradição, permitiu a análise do dinamismo, da provisoriedade e das transformações implícitas no cotidiano de crianças e adolescentes com MMC e sua família.

Os participantes do estudo foram 16 famílias de crianças e adolescentes com MMC (16 
mães e 16 crianças e adolescentes com MMC, num total de 32 participantes), que já haviam realizado o programa de reabilitação em um hospital de referência, em Belo Horizonte/MG. Os critérios de inclusão foram famílias de crianças e adolescentes com idade entre cinco e 18 anos que participaram do programa de reabilitação entre os anos de 2010 e 2015. Reconhecendo-se a continuidade do cuidado no domicílio, como um processo que é construído de forma gradual e, considerando-se as dificuldades enfrentadas pela família, foi estabelecido, como critério de inclusão, o período de cinco anos de acompanhamento da criança ou do adolescente pela instituição. Os critérios de exclusão incluíram famílias de crianças e adolescentes com problemas agudos, relatados no momento do convite, mudança de endereço ou admitidos em outras instituições durante o período de coleta de dados. Por buscar compreender as vivências das famílias, crianças institucionalizadas não foram incluídas.

Para a definição dos participantes, estimou-se o agendamento de atendimentos na instituição no mês de maio de 2015. No período, havia 54 atendimentos previstos de crianças e de adolescentes com MMC. Após consulta a esses prontuários, identificaram-se 26 potenciais participantes, uma vez que foram excluídos 28 prontuários de indivíduos que não atendiam aos critérios de inclusão. Foi estabelecido contato via telefone com as mães dos 26 potenciais participantes e 10 optaram por não participarem.

A coleta de dados ocorreu no período de maio a agosto de 2015. Foram utilizados dois instrumentos de pesquisa: a entrevista semiestruturada e a observação, ambas guiadas por roteiro. Utilizou-se diário de campo, para o registro das observações, que foram realizadas, durante as três visitas domiciliares, a cada família participante do estudo. As visitas eram realizadas com intervalo de trinta dias. A entrevista foi gravada, após a autorização dos participantes e realizadas sempre, na primeira visita domiciliar, com duração média de 45 minutos e tempo total de 10 horas e 36 minutos e 123 páginas. O roteiro de entrevista semiestruturado foi elaborado pela pesquisadora principal, utilizando-se de sua experiência profissional no atendimento às crianças, a literatura pertinente e o referencial teórico. A realização das visitas domiciliares permitiu a observação de atividades diárias dos participantes e a interação, por meio de escuta ativa, diálogo, percepções acerca da realidade cotidiana, que culminavam em orientação de condutas e na realização de cuidados. 0 tempo total das observações foi de 52 horas e 37 minutos, com tempo médio de uma hora e oito minutos, por visita.

O material obtido das entrevistas e das observações foi explorado, valendo-se da técnica da Análise de Conteúdo Temática, que utiliza as fases de pré-análise; análise do material; e tratamento dos resultados, para contemplação o método ${ }^{(17)}$.

Na pré-análise, foi realizada a transcrição e codificação das entrevistas e das observações. As entrevistas foram codificadas com caracteres alfanuméricos, nomeadamente E1 a E16, referentes à ordem em que foram realizadas. A letra " $M$ " foi adicionada à codificação correspondente à entrevista, quando se tratou de um relato da mãe, e a letra " $C$ " corresponde à criança ou ao adolescente com MMC. Para a análise, foi feita a leitura em profundidade de todo o material disponível e os procedimentos de recorte, classificação e agregação das unidades de sentido. A análise temática busca descobrir os núcleos de sentido de uma comunicação, cuja presença ou frequência signifique alguma coisa para o objeto analítico estudado ${ }^{(18)}$.

Utilizou-se dessa orientação, para a elaboração das unidades de sentido, que foram selecionadas, com base em sua relação com o objetivo do estudo e com as categorias analíticas da continuidade do cuidado e do cotidiano, sendo construída a categoria empírica: "Vivências familiares e o custo do cuidado".

O convite, para a participação no estudo era, a princípio, feito à mãe da criança ou do adolescente, via telefone. Nesse momento, era explicado que o convite se estendia ao filho, sendo ele um participante do estudo e mencionada a necessidade de assinatura de documento legal por ambos. Na primeira visita domiciliar, os objetivos eram reforçados, considerando-se a possibilidade de desistência e era solicitada a assinatura no Termo de Consentimento Livre e Esclarecido (TCLE), pela mãe e, no Termo de Assentimento Livre e Esclarecido (TALE), pelo participante menor de idade.

Os aspectos éticos foram observados conforme especificado na Resolução 466/2012 do Conselho Nacional de Saúde ${ }^{(19)}$. O projeto foi aprovado pelo comitê de ética em pesquisa da Universidade Federal de Minas Gerais (UFMG), sob o parecer número 1.036 .625 e pelo comitê de 
ética da rede de hospitais de reabilitação, número 1.089 .123 , ambos de 2015.

\section{RESULTADOS E DISCUSSÃO}

As mães participantes do estudo tinham entre 26 e 52 anos de idade, com média de 37 anos. Apesar de encontrarem-se em idade produtiva, apenas duas mulheres trabalhavam fora do domicílio. A maioria das participantes, 14 mães, tinham trabalho formal, antes do nascimento da criança com MMC, evidenciando que as suas necessidades físicas e emocionais exigem esforço, dedicação e tempo materno.

Quanto à escolaridade, três mulheres declararam possuir o ensino médio incompleto; seis, o ensino médio completo; seis mães declararam possuir o ensino fundamental incompleto e uma declarou ser iletrada. Duas mães haviam voltado a estudar recentemente e estavam cursando o ensino superior. Observou-se que doze mulheres eram casadas, dentre as quais três relataram que os maridos estavam desempregados no momento da pesquisa.

A construção histórica e social do papel da mulher na sociedade reflete-se na organização familiar, especialmente, após o nascimento de uma criança com $\mathrm{MMC}$, determinando a frequência de mães como cuidadoras, sendo quem geralmente abdicam de seus projetos de vida e objetivos para exercer esse novo papel ${ }^{(11)}$.

"Depois que ela nasceu eu não tive mais condições de trabalhar. E eu prefiro cuidar do que deixar para uma outra pessoa" (M.E.16).

"Eu tive que vir para $\mathrm{BH}$ porque os tratamentos dela são todos aqui... e lá não tem recurso nenhum, até hoje. Mudei para cá por conta dela. E parei de trabalhar também" (M.E.10).

$O$ custo indireto, provocado pela perda da capacidade laboral materna, que deixa de possuir uma fonte de renda, acarreta, ainda, desgaste psicológico e emocional, pela perda de papel ou função social, provocando também um custo intangível ${ }^{(13-20)}$. Abdicar de um emprego fora do domicílio, também, foi um Quadro 1 - Uso e acesso a materiais e medicamentos relacionados ao cuidado de crianças e adolescentes com mielomeningocele. Belo Horizonte, MG, Brasil, 2015.

\begin{tabular}{|l|c|c|c|}
\hline \multicolumn{1}{|c|}{ MATERIAL } & \multicolumn{2}{c|}{ USO REGULAR } & USO IRREGULAR OU NÃO USO \\
\hline Medicação & COMPRA & ADQUIRE & 2 \\
\hline Fraldas & 12 & 2 & 2 \\
\hline Cateter Urinário Descartável & 13 & 1 & $1^{*}$ \\
\hline Luvas & 6 & 9 & 6 \\
\hline
\end{tabular}

Elaborado pelos autores * Uma adolescente faz uso de sonda metálica. aspecto identificado em estudo com mães cuidadoras de crianças com paralisia cerebral, que afirmaram dificuldade em conseguir deixá-las sob a responsabilidade de outras pessoas, propiciando uma ruptura de sua própria vida pessoal e profissional e uma obrigatoriedade no cuidar ${ }^{(21)}$.

As crianças e adolescentes com mielomeningocele tinham entre 7 e 17 anos, com média de 12 anos de idade; 13 eram do sexo feminino e três do sexo masculino. Apenas três participantes, todas do sexo feminino, eram totalmente independentes, para o autocuidado e atividades de vida diária; dois eram totalmente dependentes de cuidados de terceiros, inclusive, para a alimentação e 11 participantes apresentavam dependência parcial para o autocuidado e atividades de vida diária.

Quanto ao uso de medicação, 14 faziam uso regular de, no mínimo, um medicamento, entre os quais dois os adquirem gratuitamente, ao passo que 12 compram, pelo menos, parte da medicação usada regularmente. Em virtude da incontinência vesical e intestinal, o uso de fraldas se faz necessário e regular para 14 crianças e adolescentes sendo que 13 necessitam comprá-las.

Todos os participantes fazem uso de cateter urinário, para o esvaziamento da bexiga, apenas uma adolescente utilizava sonda metálica. Quanto à aquisição do cateter urinário descartável, seis famílias relataram não conseguir adquirir esse material de forma gratuita.

Para a realização de medidas de reeducação intestinal, as famílias necessitam de luvas descartáveis, sendo seu uso irregular entre os participantes. Foi identificado que quatro famílias compram esse material e seis adquirem luvas descartáveis, gratuitamente, nas unidades de saúde de referência.

O Quadro 1 ilustra o uso regular ou não de materiais e medicamentos necessários ao cuidado de crianças e adolescentes com MMC, bem como sua forma de acesso, se por compra ou aquisição junto à rede pública de serviços de saúde. 
$\mathrm{O}$ cuidado à criança e ao adolescente com MMC em domicílio tem um custo não apenas emocional, para a família, mas também financeiro. Esse custo financeiro direto é agravado pela cronicidade, que exige gastos contínuos e crescentes, uma vez que, com o crescimento e o desenvolvimento da criança, modificam-se suas necessidades, com mudança de seus aparatos de suporte, medicamentos e materiais para a realização de cuidados. As demandas de cuidados prioritárias, para as crianças e adolescentes com MMC, exigem o uso frequente de medicamentos, fraldas, cateter urinário e luvas descartáveis. As famílias manifestaram dificuldade na aquisição de materiais e medicamentos junto à rede básica de serviços de saúde, que ocorreu de forma irregular, evidenciando descontinuidade dos serviços de atenção básica no atendimento a essa demanda.

A rede de serviços da Atenção Básica ou Atenção Primária à Saúde representa a entrada do usuário ao sistema de saúde, prevê a oferta de insumos e medicamentos básicos para o cuidado e deve realizar atendimento com base nos atributos essenciais de acesso, longitudinalidade, integralidade e atenção centrada na família, respeitando os princípios doutrinários do Sistema Único de Saúde (SUS) da integralidade, universalidade e equidade ${ }^{(22)}$. No entanto as mães destacaram dificuldades quanto à aquisição de material e dificuldades de atenção ao filho com condição crônica.

"A fralda também eu estava ganhando. E não sei o que aconteceu que eles pararam de doar a fralda. Desde o mês passado. Eles estavam doando um pacote de fralda por mês com 10 fraldas. $O$ resto era tudo comprado. Um pacote com 10 não dá nem para uma semana" (M.E.8).

"Eu ganho sonda, mas sempre está em falta. Quando falta a gente compra" (M.E.13).

A aquisição de materiais de forma gratuita representou uma dificuldade mencionada por todas as famílias que se veem obrigadas a comprar insumos que deveriam ser fornecidos. A baixa renda e a necessidade de compra de materiais comprometem o orçamento das famílias, limitando, inclusive, suas atividades sociais e de lazer.

Os gastos financeiros com as crianças e os adolescentes em condição crônica atingem famílias de diferentes níveis socioeconômicos e de diferentes formas, $e$ as dificuldades financeiras, decorrentes do tratamento associadas à alta carga de cuidados, podem estar relacionadas à maior probabilidade de sintomas depressivos nos pais ${ }^{(23)}$.

A revisão de literatura acerca da necessidade de suporte aos pais de crianças com câncer, pelo impacto psicológico, evidencia a necessidade imperativa de avaliação e acompanhamento contínuos de sua saúde mental pelo risco de desajuste parental, relacionado a fatores como baixo nível socioeconômico e renda familiar, menor nível de escolaridade, desemprego e risco e gravidade da doença da criança ${ }^{(24)}$.

A renda familiar média declarada de, aproximadamente, dois salários mínimos (salário mínimo à época, $\mathrm{R} \$ \mathbf{7 8 8 , 0 0}$ ), o baixo nível de escolaridade materna e o desemprego parental expõem as famílias participantes do estudo ao risco de desordens emocionais e psicológicas. Ressalta-se que 12 famílias recebiam o Benefício da Prestação Continuada (BPC), no valor de um salário mínimo vigente, garantido pelo governo federal às pessoas idosas, com condições crônicas e de baixa renda comprovada e apenas duas possuíam o benefício como fonte de renda.

A revisão bibliográfica, que analisou a produção do conhecimento acerca das condições crônicas ou doenças crônicas de crianças e adolescentes, também, identificou o baixo nível socioeconômico agravado pelas dificuldades de aquisição de materiais e insumos, em serviços de saúde, como um importante desafio para as famílias ${ }^{(25)}$.

A fragilidade clínica inerente aos participantes demanda a necessidade de acompanhamento regular por serviços e equipes de saúde especializados. O deslocamento até os serviços de referência representa um custo direto mencionado por metade das famílias que participaram deste estudo. Os gastos com transporte representam uma dificuldade relatada por famílias residentes, também, na cidade de Belo Horizonte, não sendo um gasto exclusivo para famílias de outras cidades e que precisam de deslocamentos maiores, evidenciando o desafio social relacionado à mobilidade e à acessibilidade de crianças com deficiência.

"Na verdade, o que eu gasto com ele é questão de gasolina, muita. Porque tem que buscar a fralda, tem que ir ao hospital, às vezes tem que ir na escola. Então assim, gasolina a gente gasta muita" (M.E.5) 
"Tem que colocar gasolina no carro para ir. Às vezes quando não é meu marido que leva tem que pagar alguém, pagando a gasolina da pessoa que vai me levar" (M.E.9).

Em pesquisa que avaliou a experiência financeira de famílias no cuidado às crianças e adolescentes com câncer, realizada em município do estado de São Paulo, Brasil, demonstrou que o aumento dos gastos ocorreu, majoritariamente, com alimentação, transporte, aquisição de materiais não concedidos por serviços de atenção básica e necessidade de reformas estruturais no domicílio ${ }^{(26)}$.

As famílias das três adolescentes (E.1, E.4 e E.7) independentes apresentaram custos diretos mensais menores, quando comparadas aos demais participantes do estudo. A família E.1 relatou custo direto com a compra de cateter urinário para realização de cateterismo vesical intermitente limpo. A família E.4 declarou custo direto com medicamentos e fraldas de, aproximadamente, $\mathrm{R} \$ 230,00$ mensais. A família de E.7 relatou custo direto com a compra de gel lubrificante, para o cateter urinário e com transporte, uma vez que a adolescente faz uso de sonda metálica, e a medicação é adquirida na rede básica de serviços de saúde. As famílias das duas crianças, totalmente dependentes (E.10 e E.16), que possuíam grave comprometimento cognitivo e faziam uso de um maior número de medicações, informaram custo direto mensal médio de $\mathrm{R} \$ 550,00$. Os demais participantes relataram possuir custo mensal médio de $\mathrm{R} \$$ 290,00.

Os custos intangíveis ou emocionais são imensuráveis e relacionam-se à dor e ao sofrimento que provocam repercussões emocionais e psicológicas, comprometendo a qualidade de vida de indivíduos acometidos por doença ou condições crônicas e seus cuidadores $^{(13-14)}$. Trata-se de elementos subjetivos e de difícil avaliação, talvez por isso, pouco explorados na literatura nacional.

"Dá vontade até de chorar quando não tem... para comprar as coisas... não é fácil..." (M.E.3).

Crianças e adolescentes com MMC necessitam de cuidados não habituais contínuos, durante toda a vida, podem enfrentar intercorrências clínicas que requerem longos períodos de internação e precisam de acompanhamento por serviços de saúde de forma sistemática. Tais aspectos interferem na socialização dessas crianças e adolescentes, pela necessidade de absenteísmo escolar, inviabilizando sua convivência com pares de idade, o que pode acarretar dificuldade de interação e isolamento social. A qualidade de vida de crianças e adolescentes com MMC e suas famílias pode ser comprometida de forma significativa, refletindo um custo intangível de grande magnitude.

A vulnerabilidade de crianças com deficiência não está relacionada à sua incapacidade em si. Pelo contrário, diz respeito à sua capacidade de adaptação, para a superação de fatores de risco, sejam eles físicos ou sociais. Em uma sociedade pouco inclusiva, essa adaptação é limitada, e a vulnerabilidade relaciona-se às dificuldades de inclusão social, à fragilidade clínica, ao convívio social restrito, à dificuldade de acesso aos serviços pela desarticulação do sistema e ao cuidado familiar solitário ${ }^{(27)}$.

Destaca-se, portanto o papel do enfermeiro não apenas no cuidado a crianças com MMC, mas também no estabelecendo do elo com a família, para a transição dos cuidados para a adolescência e para a vida adulta, para o enfrentamento de situações de vulnerabilidade nos diferentes ciclos da vida e para a busca de inclusão social e melhor qualidade de vida(28).

\section{CONSIDERAÇÕES FINAIS}

As vivências biopsicossociais que envolvem o cuidado de crianças e adolescentes com MMC em domicílio têm um custo não apenas emocional para a família, mas também financeiro, que é agravado pela cronicidade. O custo direto do cuidado envolve a necessidade de compra de materiais, bem como gastos com transporte, pela necessidade de deslocamentos. Os custos diretos, para indivíduos independentes, são menores quando comparados aos custos diretos de crianças e adolescentes totalmente dependentes.

Os custos indiretos do cuidado relacionamse à necessidade da mãe abdicar do próprio emprego e assumir a função de cuidadora, fato que compromete o orçamento familiar e gera desgaste emocional. $\mathrm{O}$ desgaste emocional, vivenciado pelas famílias, foi exacerbado pela demanda financeira gerada pelos cuidados, evidenciando um custo intangível.

Os profissionais devem considerar os aspectos sociais e econômicos das famílias, na elaboração do plano de cuidados, buscando garantir a oferta adequada dos insumos 
indispensáveis e elaborar estratégias que minimizem custos evitáveis.

Identificou-se que as famílias necessitam de suporte dos serviços de saúde e apresentam dificuldade de aquisição de materiais junto à Rede de Atenção Básica de Saúde, no entanto o estudo não buscou a rede de apoio social ou de assistência social, podendo ser considerada uma limitação. Reconhece-se a necessidade de pesquisas que aprofundem quanto à relação da rede de serviços, bem como a elaboração de estudos que envolvem o custo para o cuidado de crianças com condições crônicas. Sugere-se a efetiva integração, nos âmbitos da educação, saúde e assistência social, para a construção de políticas públicas direcionadas.

\section{REFERÊNCIAS}

1- Mohd-Zin SW, Marwan Al, Abou Chaar MK, Ahmad-Annuar A, Abdul-Aziz NM. Spina bifida: Pathogenesis, mechanisms, and genes in mice and humans. Scientifica 2017;5364827. DOI: $10.1155 / 2017 / 5364827$

2- Copp AJ, Greene NDE. Neural tube defects: Disorders of neurulation and related embryonic processes. Wiley Interdiscip Rev Dev Biol. 2013;2(2):213-27. DOI: 10.1002/wdev.71

3- Jiménez-León JC, Batancourt-Fursow YM, Jiménez-Betancourt CS. Malformaciones del sistema nervioso central: Correlación neuroquirúrgica. Rev Neurol. 2013;57(Supll 1):37-45. DOI: $10.33588 / \mathrm{rn} .57501 .2013223$

4- Oakeshott $P$, Poulton A, Hunt GM, Reid F. Walking and living independently with spina bifida: A 50-year prospective cohort study. Dev Med Child Neurol. 2019;61(10):1202-07. DOI: 10.1111/dmcn.14168

5- Adzick N, Thom EA, Spong CY, Brock JW, Burrows $\mathrm{PK}$, Johnson MP, et al. A randomized trial of prenatal versus postnatal repais of myelomeningocele. N Engl J Med. 2011;364(11):993-1004. DOI: 10.1056/NEJMoa1014379

6- Gaiva MA, Corrêa E, Espírito Santo E. Perfil clínico-epidemiológico de crianças e adolescentes que vivem e convivem com espinha bífida. Rev Bras Crescimento Desenvolv Hum. 2011;21(1):99110. DOI: $10.7322 /$ ihgd.19999

7- Faria TCC, Cavalheiro S, Hisaba WJ, Moron AF, Torloni MR, Oliveira AL. Improvement of motor function and decreased need for postnatal shunting in children who had undergone intrauterine myelomeningocele repair. Arq Neuropsiquiatr. 2013;71(9A):604-8. DOI: $\underline{10.1590 / 0004-282 \times 20130104}$
8- Ramsundhar N, Donald K. An approach to the developmental and cognitive profile of the child with spina bifida. SAMJ, S Afr Med. 2014;104(3):1-3. DOI: 10.7196/samj.8048

9- Faleiros F., Pelosi G, Warschausky S, Tate D, Käppler $\mathrm{C}$, Thomas E. Factors influencing the use of intermittent bladder catheterization by individuals with spina bifida in Brazil and Germany. Rehabil Nurs. 2018;43(1):46-51. DOI: 10.1002/rnj.302

10- Szymanski KM, Cain MP, Whittam B, Kaefer $M$, Rink RC, Misseri R. All incontinence is not created equal: Impact of urinary and fecal incontinence on quality of life in adults with spina bifida. J Urol. 2017;197(3):885-91. DOI: 10.1016/j.juro.2016.08.117

11- Freitas GL, Sena RR, Silva JCR, Faleiros-Castro F. Reabilitação de crianças e adolescentes com mielomeningocele: $O$ cotidiano de mães cuidadoras. Rev Gaúcha Enferm. 2016;37(4):1-8. DOI: 10.1590/1983-1447.2016.04.60310

12- Faleiros F, Toledo C, Gomide MFS, Faleiros RG, Käppler C. Right to health care and materials required for intermittent catheterization: A comparison between Germany and Brazil. Quality Primary Care 2015 [citado em 13 out 2017]; 23(3):127-33. Disponível em: http://primarycare.imedpub.com/right-to-healthcare-and-materials-requiredfor-intermittentatheterization-a-comparisonbetween-germanyand-brazil.pdf

13- Aung L, Saw SM, Chan MY, Khaing T, Quah TC, Verkooijen HM. The hidden impact of childhood cancer on the family: A multiinstitutional study from Singapore. Ann Acad Med Singapore [citado em 25 jun 2017]; 41(4):170-5. Disponível em: http://www.annals.edu.sg/pdf/41VolNo4Apr2012 N41N4p170.pdf

14- Rascati KL. Introdução à farmacoeconomia. Porto Alegre: Artmed; 2010.

15- Gadotti M. Concepção da dialética da educação: Um estudo introdutório. 9a ed. São Paulo: Cortez; 1995.

16- Heller A. O cotidiano e a história. São Paulo: Terra e Paz; 2008.

17- Bardin L. Análise de conteúdo. 4a ed. Revista e atualizada. Lisboa: Edições 70; 2009.

18- Minayo MCS. O desafio do conhecimento: Pesquisa qualitativa em saúde. 12a ed. São Paulo: Hucitec; 2010.

19- Brasil. Ministério da Saúde. Resolução CNS/MS no 466, de 12 de dezembro de 2012. Diário Oficial da União 2012. Disponível em: 
http://bvsms.saude.gov.br/bvs/saudelegis/cns/20 13/res0466 $1212 \quad 2012 . \mathrm{html}$

20- Wijesinghe CJ, Fonseka P, Hewage CG. The development and validation of an instrument to assess caregiver burden in cerebral palsy: Caregiver Difficulties Scale. Ceylon Med J. 2013;58(4):162-7. DOI: 10.4038/cmj.v58i4.5617

21- Dezoti AP, Alexandre AMC, Freire MHS, Mercês NNA, Mazza VA. Apoio social a famílias de crianças com paralisia cerebral. Acta Paul Enferm. 2015;28(2). DOI: 10.1590/1982-0194201500029

22- 22 Carnut L. Cuidado, integralidade e atenção primária: Articulação essencial para refletir sobre o setor saúde no Brasil. Saúde Debate 2017;41(115):1177-86. DOI: 10.1590/0103-1104201711515

23- Creswell PD, Wisk LE, Litzelman K, Allchin A, Witt WP. Parental depressive symptoms and childhood cancer: The importance of financial difficulties. Support Care Câncer 2014;22(2):50311. DOI: $10.1007 / \mathrm{s} 00520-013-2003-4$

24- Kearney JA, Salley CG, Muriel AC. Standards of psychosocial care for parents of children with cancer. Pediatr Blood Cancer 2015;62 (Suppl 5):632-83. DOI: $10.1002 /$ pbc.25761

25- Moreira MCN, Gomes R, SÁ MRC. Doenças crônicas em crianças e adolescentes: Uma revisão bibliográfica. Ciênc. Saúde Coletiva 2014;19(7):2083-94. DOI: 10.1590/141381232014197.20122013

26- Andrade RC, Marques AR, Leite ACAB, Martimiano RR, Santos $B D$, Pan $R$, et al. Necessidades dos pais de crianças hospitalizadas: Evidências para o cuidado Rev Eletr Enf. 2015;17(2):379-94. DOI: 10.5216/ree.v17i2.30041 27- Silveira A, Neves ET. Vulnerabilidade das crianças com necessidades especiais de saúde: Implicações para a enfermagem. Rev Gaúcha Enferm. 2012;33(4):172-80. DOI: 10.1590/S198314472012000400022

28- Malheiro MIDC, Gaspar MF, Barros L. Programa de educação para a autogestão em adolescentes com espinha bífida: o que dizem os jovens e seus cuidadores. Cogitare Enferm. 2019;24:1-12. DOI: $10.5380 / c e . v 24 i 0.62222$

Nota: Artigo extraído da Tese de Doutorado: "A (des)continuidade do cuidado de crianças e adolescentes com mielomeningocele no domicílio", vinculada ao Programa de Pós-Graduação em Enfermagem da Universidade Federal de Minas Gerais.

\section{Agradecimentos}

À Dra Roseni Rosângela de Sena (in memoriam), professora Emérita da Escola de Enfermagem da Universidade Federal de Minas Gerais, pela orientação, dedicação e apoio para a concretização deste projeto.

Recebido em: 10/02/2019

Aprovado em: 18/09/2019

\section{Endereço de correspondência:}

Giselle Lima de Freitas

Avenida Alfredo Balena, 190. Sala 400. Santa Efigênia

CEP: 30130-100 - Belo Horizonte/MG - Brasil

E- mail: gisellelf@yahoo.com.br 